\title{
A Structural Analysis of Curve Deformation by Discontinuous Transformations
}

\author{
Hirobımi Nishida \\ Ricoh Information and Communication R\&D Center \\ 3-2-3 Shin-Yokohama \\ Kohoku-ku, Yokohama 222, Japan
}

\begin{abstract}
Structural analysis and description depend on the topological structure of the shape, and therefore, they are sensitive to discontinuous transformations which change the topology of the shape. In this paper, we give a complete analysis of the a priori effects of some commonly occurring discontinuous transformations. Some systematic transformation laws have been obtained based on the shape description in terms of quasi-convexity/concavity and quantized directional features. We mention some applications of the analysis.
\end{abstract}

\section{Introduction}

Shape description has been an important research subject in image analysis, computer graphics, and pattern recognition. The criteria and feature selection for shape description depend on the particular applications. In particular, handwriting recognition has been a main theme in both theory and practice of pattern recognition. A prime difficulty of handwriting recognition is the variety of shape deformations and it leads to some unique requirements for the shape description as follows:

- The shape deformations of handwritten characters are elastic, highly nonlinear, and therefore, difficult to analyze. For describing such complex objects, some global, qualitative, structural features are appropriate rather than local, quantitative descriptions based on some analytical or statistical shape models.

- Such discontinuous deformations as caused by stroke connections are unique to handwritten characters. The shape description must be robust against and accommodate such deformations so that various deformed patterns can be represented by a small number of classes.

Throughout more than a quarter of a century of researches, it has been found that such features as quasi-topological features (convexity, concavity, and loop), quantized directional features, and singularities (branch points and crossings) are effective and powerful for the shape description of handwritten characters. Based on this observation, Nishida and Mori [5], Nishida [1], [2] presented a clear, rigorous method for structural description and feature extraction of thinline pictures in terms of global, qualitative features. Shapes can be described 
by a few components along with rich features and relations among components, and the description is robust against certain types of continuous deformation.

Exploring methods for shape description and recognition robust against such deformations as caused by stroke connections has been a challenging problem. In order to overcome the difficulties in handwriting recognition, there have been some works conducted for analyzing and modeling the shape deformation of unconstrained handwritten characters. The deformations can be generalized into the two types of transformations as follows:

T1: concatenating two end points of curve components by moving them slightly so that the two points coincide (Fig. 2).

T2: connecting two end points of curve components with an additional curve (Fig. 3).

These deformations are discontinuous and change the topological structure of the shape completely. Structural shape descriptions are, in general, based on the topological structure of the shape, and we need to address these types of shape transformations in order to explore methods for shape description and recognition robust against such deformations as caused by stroke connections. A first step for overcoming the difficulties is to carry out a systematic, complete analysis of the a priori effects of these commonly occurring discontinuous transformations, leading to a small, tractable number of distinct cases. Once we have a complete, a priori knowledge of all possible cases, we can analyze all instances satisfying certain realistic conditions in a unified, systematic way withotht resorting to heuristics.

This paper is organized as follows: In Section 2, we mention the outline of the structural analysis of curves. In Section 3, we give a complete analysis of the a priori effects of commonly occurring discontinuous transformations $\mathrm{T} 1$ and T2. In Section 4, we describe some applications of the analysis. Section 5 is the conclusion.

\section{Structural Analysis of Curves}

In this section, we review the structural analysis and description of curves by $2 N$-directional features ( $N$ is a natural number) and quasi-convexity/concavity. When the curve has singular points, whose order is three or more, the curve is transformed into components which are topologically equivalent to line segments or circles by singular point decomposition [5]. Therefore, we assume that the curve is a simple (open) arc or a simple closed curve without a loss of generality. For the details of the analysis and description, refer to Nishida and Mori [5], Nishida [1], [2].

On a two dimensional plane, we introduce $N$-axes together with $2 N$ direction codes. For instance, when $N=4$, eight directions are defined along with the four axes as shown in Fig. 1(a). Based on these $N$-axes together with $2 N$ direction codes, the analysis is performed hierarchically in the following way: First, the curve is decomposed into primitives and adjacent primitives are concatenated. 


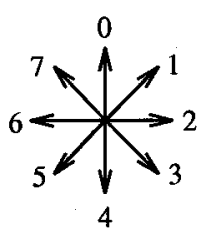

(a)

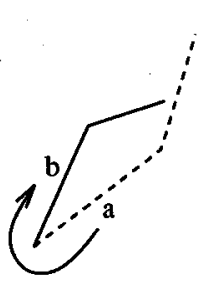

(b)

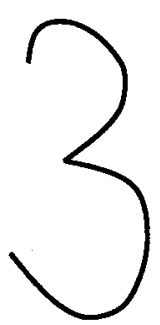

(c)

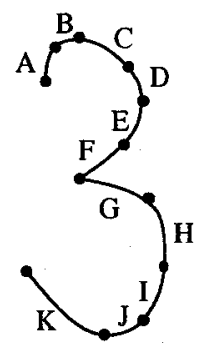

(d)

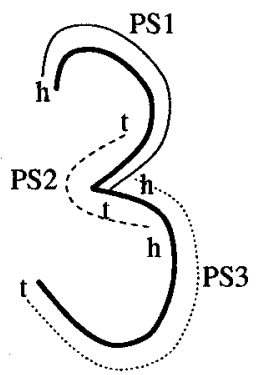

(e)

Fig. 1. Structural analysis of a curve.

The concatemation is classified according to the direction of convexity. Primitive sequences are generated by linking the concatenations of primitives. A label is given to each primitive sequence according to the properties of the primitives and their concatenations forming the sequence. Two types of connection are introduced to primitive sequences. The structure of a curve is described by a string of primitive sequences and connections.

A curve is decomposed into primitives at extremal points along each of the $N$-axes. For adjacent primitives $a$ and $b$, we write a concatenation of primitives $a$ and $b$ as $a \stackrel{j, k}{\longrightarrow} b$. The arrow " $\rightarrow$ " means that the primitive $a$ is concatenated to $b$ so that we turn to right when traversing them from $a$ to $b$ (see Fig. 1(b)), and " $j, k$ " denotes the direction codes of convexity formed by $a$ and $b$.

By linking the concatenations of primitives, the primitive sequence is constructed:

$$
a_{0} \stackrel{j(1,0), j(1,1)}{\longrightarrow} a_{1} \stackrel{j(2,0), j(2,1)}{\longrightarrow} \cdots \stackrel{j(n, 0), j(n, 1)}{\longrightarrow} a_{n} .
$$

The label of the primitive sequence, PS-label for short, $\langle$ rot, idr $\rangle$ is given to the sequence (1) composed of $n+1$ primitives by the following formulae:

$$
\begin{gathered}
i d r=j(1,0) \\
\operatorname{rot}=\sum_{i=1}^{n}\{(j(i, 1)-j(i, 0)) \%(2 N)\}+\sum_{i=1}^{n-1}\{(j(i+1,0)-j(i, 1)) \%(2 N)\}+2
\end{gathered}
$$

rot and $i d r$ represent the rotation number and the initial direction of rotation quantized in $2 N$ directions. Furthermore, the two end points of the primitive sequence are called $h$-point (on $a_{0}$ ) and $t$-point (on $a_{n}$ ).

Two primitive sequences are connected to one another by sharing the first primitive (h-connection) or the last primitive (t-connection). For two adjacent primitive sequences $e_{0}$ and $e_{1}$, the connections are denoted by $e_{0} \frac{h}{e_{1}}$ and $e_{0} \underline{t} e_{1}$ respectively. 
Example 1: Fig. 1(d) illustrates the decomposition of a line-picture shown in Fig. 1(c) into primitives when $N=4$. We obtain the following concatenations for these primitives.

$$
\begin{aligned}
& A \stackrel{7,7}{\longrightarrow} B, B \stackrel{0,0}{\longrightarrow} C, C \stackrel{1,1}{\longrightarrow} D, D \stackrel{2,2}{\longrightarrow} E, E \stackrel{3,3}{\longrightarrow} F, \\
& G \stackrel{5,7}{\longrightarrow} F, G \stackrel{1,1}{\longrightarrow} H, H \stackrel{2,2}{\longrightarrow} I, I \stackrel{3,3}{\longrightarrow} J, J \stackrel{4,4}{\longrightarrow} K .
\end{aligned}
$$

Then, the following primitive sequences are generated (see Fig. 1(e)):

$$
\begin{array}{r}
P S_{1}: A \stackrel{7,7}{\longrightarrow} B \stackrel{0,0}{\longrightarrow} C \stackrel{1,1}{\longrightarrow} D \stackrel{2,2}{\longrightarrow} E \stackrel{3,3}{\rightarrow} F\langle 6,7\rangle, \\
P S_{2}: G \stackrel{5,7}{\longrightarrow} F\langle 4,5\rangle, \quad P S_{3}: G \stackrel{1,1}{\longrightarrow} H \stackrel{2,2}{\longrightarrow} I \stackrel{3,3}{\longrightarrow} J \stackrel{4,4}{\longrightarrow} K\langle 5,1\rangle,
\end{array}
$$

Since the primitive sequences $P S_{1}, P S_{2}$, and $P S_{3}$ are connected as $P S_{1} \underline{t} P S_{2}$, $P S_{2} \stackrel{h}{P} P S_{3}$, the line-picture shown in Fig. $1(\mathrm{c})$ is described as $\langle 6,7\rangle^{\underline{t}}\langle 4,5\rangle \underline{h}\langle 5,1\rangle$.

\section{Curve Deformation by Discontinuous Transformations}

The structural descriptions based on the primitive sequences depend on the topological structure of the curve, which is changed completely by discontinuous transformation $\mathrm{T} 1$ and $\mathrm{T} 2$. In this section, we systematically analyze how the features and structure of the curve are transformed by various types of deformations belonging to $\mathrm{T} 1$ and $\mathrm{T} 2$. Becanse of the space limitation, we show only some main results of the analysis in this paper. The result of a complete and exhaustive analysis is given in Nishida [4]. Throughout this section, suppose that there are two primitive sequences $p_{0}$ and $p_{1}$ with PS-labels $\left\langle r_{0}, d_{0}\right\rangle$ and $\left\langle r_{1}, d_{1}\right\rangle$ $\left(r_{0}, r_{1} \geq 2\right)$, respectively.

\subsection{Structural Transformation by Concatenating End Points}

We analyze the structural transformation caused by each operation of $\mathrm{T} 1$, i.e., concatenating two end points of primitive sequences so that the two end points coincide.

Theorem 1: If t-point of $p_{0}$ and h-point of $p_{1}$ are concatenated, then the local structure of the curve is transformed as follows:

- If $k \equiv\left(d_{1}-r_{0}-d_{0}\right) \%(2 N)=2 N-1$ or $0 \leq k \leq N-1$, then the primitive sequences $p_{0}$ and $p_{1}$ can be merged into one primitive sequence $P_{0}$ with PSlabel $\left\langle r_{0}+r_{1}-2+(k+2) \%(2 N), d_{0}\right\rangle .(a \% b$ is the residue when $a$ is divided by $b>0$.)

- If $N-1 \leq k \leq 2 N-2$, then a new primitive sequence can be created and the local structure of the curve can be transformed to $P_{0} \underline{t} P_{1} \underline{h} P_{2}$, where PS-labels of the primitive sequences are $P_{0}:\left\langle r_{0}, d_{0}\right\rangle, P_{1}:\langle 2+(-k-$ 2)\%(2N), $\left.\left(d_{1}+N\right) \%(2 N)\right\rangle, P_{2}:\left\langle r_{1}, d_{1}\right\rangle$. The primitive sequence $P_{0}$ and $P_{2}$ correspond to $p_{0}$ and $p_{1}$ respectively. 
Example 2: When $k=N-1$, the curve structure can be transformed in two ways according to the configuration of two primitive sequences. For instance, when $N=4$ (eight directions), if PS-labels of $p_{0}$ (left of Fig. 2(a)) and $p_{1}$ (right) are $\langle 5,0\rangle$ and $\langle 2,0\rangle(k=(0-5-0) \% 8=(-5) \% 8=3)$, then the local structure of the curve can be either one primitive sequence $P_{0}$ with PS-label $\langle 10,0\rangle$ (Fig. 2(b)), or $P_{0} \underline{t} P_{1} \underline{h} P_{2}$ with PS-labels $P_{0}:\langle 5,0\rangle, P_{1}:\langle 5,4\rangle$, and $P_{2}:\langle 2,0\rangle$ (Fig. $2(\mathrm{c}))$.

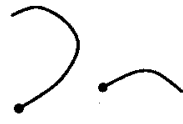

(a)

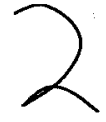

(b)

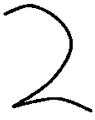

(c)

Fig. 2. When we concatenate the two end points denoted by filled circles in (a), there are two possible structures (b) and (c) according to the transformation rules.

Theorem 2: If h-point of $p_{0}$ and h-point of $p_{1}$ are concatenated, then the local structure of the curve is transformed as follows $\left(k=\left(d_{1}-d_{0}\right) \%(2 N)\right)$ :

\begin{tabular}{ccll}
\hline Condition & $M$ Structure PS-label \\
\hline \hline$N \leq k \leq 2 N-1$ or $k=0$ & 2 & $P_{0}{ }^{\underline{h}} P_{1}$ & $P_{0}:\left\langle r_{0}, d_{0}\right\rangle$ \\
& & $P_{1}:\left\langle r_{1}+(k+N) \%(2 N),\left(d_{0}+N\right) \%(2 N)\right\rangle$ \\
\hline $0 \leq k \leq N$ & 2 & $P_{0}{ }^{\underline{h}} P_{1}$ & $P_{0}:\left\langle r_{0}+(N-k) \%(2 N),\left(d_{1}+N\right) \%(2 N)\right\rangle$ \\
& & & $P_{1}:\left\langle r_{1}, d_{1}\right\rangle$ \\
\hline
\end{tabular}

$M$ is the number of primitive sequences after the two primitive sequences are concatenated, and $P_{0}$ and $P_{M-1}$ on the new curve correspond to $p_{0}$ and $p_{1}$ on the original curve. When $M \geq 3$, primitive sequences $P_{1}$ through $P_{M-2}$ do not exist on the original curve and they are introduced by the operation $\mathrm{T} 1$.

Theorem 3: If $\mathrm{t}$-point of $p_{0}$ and $\mathrm{t}$-point of $p_{1}$ are concatenated, then the local structure of the curve is transformed as follows $\left(k=\left(r_{1}+d_{1}-r_{0}-d_{0}\right) \%(2 N)\right)$ :

\begin{tabular}{cccc}
\hline Condition & \multicolumn{3}{c}{$M$ Structure PS-label } \\
\hline $0 \leq k \leq N$ & 2 & $P_{0}{ }^{t} P_{1}$ & $P_{0}:\left\langle r_{0}, d_{0}\right\rangle, P_{1}:\left\langle r_{1}+(N-k) \%(2 N), d_{1}\right\rangle$ \\
\hline$N \leq k \leq 2 N-1$ or $k=0$ & 2 & $P_{0}{ }^{t} P_{1}$ & $P_{0}:\left\langle r_{0}+(k+N) \%(2 N), d_{0}\right\rangle, P_{1}:\left\langle r_{1}, d_{1}\right\rangle$ \\
\hline
\end{tabular}

\subsection{Structural Transformation by Connecting End Points}

We analyze the structural transformation caused by each operation of T2, i.e., connecting two end points of primitive sequences with an additional primitive.

Theorem 4: If $t$-point of $p_{0}$ and h-point of $p_{1}$ are connected by an additional primitive, then the local structure of the curve is transformed as follows $(k=$ $\left.\left(d_{1}-r_{0}-d_{0}\right) \%(2 N)\right)$ : 


\begin{tabular}{|c|c|}
\hline Condition & $M$ Structure PS-label \\
\hline$k=2 N-2,2 N-1$ & $P_{0}:\left\langle r_{0}+r_{1}-2+(k+2) \%(2 N), d_{0}\right\rangle$ \\
\hline $0 \leq k \leq 2 N-1$ & $P_{0}:\left\langle r_{0}+r_{1}+k, d_{0}\right\rangle$ \\
\hline $0 \leq k \leq 2 N-1$ & $\begin{aligned} 3 P_{0}-P_{1} \underline{h} P_{2} & P_{0}:\left\langle r_{0}, d_{0}\right\rangle \\
P_{1} & :\left\langle 2+(-k-2) \%(2 N),\left(d_{1}+N\right) \%(2 N)\right\rangle, \\
P_{2} & :\left\langle r_{1}, d_{1}\right\rangle\end{aligned}$ \\
\hline $0 \leq k \leq 2 N-1$ & $\begin{aligned} 3 P_{0}-P_{1}-P_{2} & P_{0}:\left\langle r_{0}+(k+l+2) \%(2 N), d_{0}\right\rangle \\
& P_{1}:\left\langle 2+l,\left(d_{1}+N\right) \%(2 N)\right\rangle, P_{2}:\left\langle r_{1}, d_{1}\right\rangle \\
& (l=0, \ldots, N-1 ;(k+l+2) \%(2 N)=0, \ldots, N)\end{aligned}$ \\
\hline $0 \leq k \leq 2 N-1$ & $\begin{aligned} 3 P_{0}-P_{1} \frac{k}{2} P_{2} P_{0}: & \left\langle r_{0}, d_{0}\right\rangle \\
P_{1}: & \left\langle 2+l,\left(r_{0}+d_{0}+N-l-2\right) \%(2 N)\right\rangle \\
P_{2}: & \left\langle r_{1}+(k+l+2) \%(2 N)\right. \\
& \left.\left(r_{0}+d_{0}-l-2\right) \%(2 N)\right\rangle \\
(l= & 0, \ldots, N-1 ;(k+l+2) \%(2 N)=0, \ldots, N)\end{aligned}$ \\
\hline
\end{tabular}

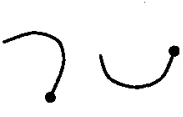

(a)

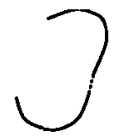

(b)

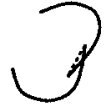

(c)

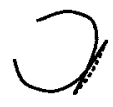

(d)

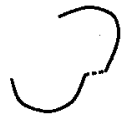

(e)

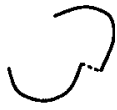

(f)<smiles>CCCCCCCCCCC</smiles>

(g)

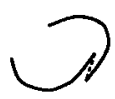

(h)

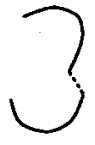

(i)

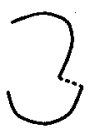

(i)

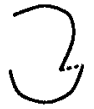

(k)

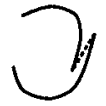

(l)

Fig. 3. When we connect the two end points denoted by filled circles in (a), with an additional primitive, there are eleven possible structures (b) through (l) according to the transformation rules.

Example 3: When $k=2 N-1$ and $N=4$, the curve structure can be transformed in eleven ways according to the configuration of two primitive sequences. For instance, if PS-labels of $p_{0}$ (left of Fig. $\left.3(\mathrm{a})\right)$ and $p_{1}$ (right) are $\langle 4,0\rangle$ and $\langle 4,3\rangle$ $(k=(3-4-0) \% 4=7)$, then the local structure of the curve can be one of the following: (1) $\langle 7,0\rangle$ (Fig. 3(b)); (2) $\langle 15,0\rangle$ (Fig. 3(c)); (3) $\langle 4,0\rangle \stackrel{\underline{t}}{\underline{1}}\langle 9,7\rangle \underline{h}\langle 4,3\rangle$ (Fig. $3(\mathrm{~d})) ;(4)\langle 5,0\rangle \underline{\underline{t}}\langle 2,7\rangle^{\underline{h}}\langle 4,3\rangle$ (Fig. $\left.3(\mathrm{e})\right) ;(5)\langle 6,0\rangle \underline{\underline{t}}\langle 3,7\rangle^{\underline{h}}\langle 4,3\rangle$ (Fig. 3(f)); (6) $\langle 7,0\rangle \underline{\underline{t}}\langle 4,7\rangle \underline{\underline{h}}\langle 4,3\rangle$ (Fig. 3(g)); (7) $\langle 8,0\rangle \underline{\underline{t}}\langle 5,7\rangle \underline{\underline{h}}\langle 4,3\rangle$ (Fig. 3(h)); (8) $\langle 4,0\rangle\rangle^{\underline{t}}\langle 2,6\rangle \underline{h}$

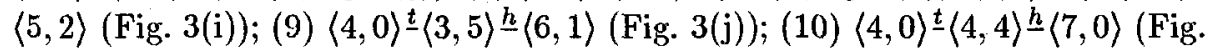
$3(\mathrm{k})) ;(11)\langle 4,0\rangle \underline{\underline{t}}\langle 5,3\rangle \underline{\underline{h}}\langle 8,7\rangle$ (Fig. $3(1)$ ).

Theorem 5: If h-point of $p_{0}$ and h-point of $p_{1}$ are connected by an additional primitive, then the local structure of the curve is transformed as follows ( $k=$ $\left.\left(d_{1}-d_{0}\right) \%(2 N)\right)$ : 


\begin{tabular}{|c|c|c|}
\hline Condition & $M$ Structure & PS-label \\
\hline$N \leq k \leq 2 N-$ & $2 P_{0} \underline{h} P_{1}$ & $\begin{array}{l}P_{0}:\left\langle r_{0}, d_{0}\right\rangle \\
P_{1}:\left\langle r_{1}+(k-N) \%(2 N),\left(d_{0}+N\right) \%(2 N)\right\rangle\end{array}$ \\
\hline $0 \leq k \leq N$ & $2 P_{0} \frac{h}{1}$ & $P_{0}:\left\langle r_{0}, d_{0}\right\rangle, P_{1}:\left\langle r_{1}+k+N,\left(d_{0}+N\right) \%(2 N)\right\rangle$ \\
\hline $0 \leq k \leq N$ & $2 P_{0} \stackrel{h}{-} P_{1}$ & $\begin{array}{l}P_{0}:\left\langle r_{0}+(N-k) \%(2 N),\left(d_{1}+N\right) \%(2 N)\right\rangle \\
P_{1}:\left\langle r_{1}, d_{1}\right\rangle\end{array}$ \\
\hline$N \leq k \leq 2 N-$ & $2 P_{0} \underline{h} P_{1}$ & $P_{0}:\left\langle r_{0}+3 N-k,\left(d_{1}+N\right) \%(2 N)\right\rangle, P_{1}:\left\langle r_{1}, d_{1}\right\rangle$ \\
\hline $0 \leq k \leq 2 N-1$ & $2 P_{0}-P_{1}$ & $\begin{array}{l}P_{0}:\left\langle r_{0}+l,\left(d_{0}-l\right) \%(2 N)\right\rangle \\
P_{1}:\left\langle r_{1}+(l+k-N) \%(2 N),\left(d_{0}-l+2\right) \%(2 N)\right\rangle \\
(l=0, \ldots, N ;(l+k-N) \% N=0, \ldots, N)\end{array}$ \\
\hline $2 N-$ & $\pm 10^{-}$ & $\begin{array}{l}P_{0}:\left\langle r_{0}, d_{0}\right\rangle, P_{1}:\left\langle 2+l,\left(d_{0}+N\right) \%(2 N)\right\rangle, \\
P_{2}:\left\langle 2+(l-k+N) \%(2 N),\left(d_{1}+N\right) \%(2 N)\right\rangle, \\
P_{3}:\left\langle r_{1}, d_{1}\right\rangle \\
(l=0, \ldots, N-1 \\
(l-k+N) \%(2 N)=0, \ldots, N-1)\end{array}$ \\
\hline
\end{tabular}

Theorem 6: If t-point of $p_{0}$ and $\mathrm{t}$-point of $p_{1}$ are connected by an additional primitive, then the local structure of the curve is transformed as follows $(k=$ $\left.\left(r_{1}+d_{1}-r_{0}-d_{0}\right) \%(2 N)\right)$ :

\begin{tabular}{clll}
\hline \multicolumn{1}{c}{ Condition } & $M$ Structure & PS-label \\
\hline \hline$N \leq k \leq 2 N-1$ & 2 & $P_{0}{ }^{t} P_{1}$ & $P_{0}:\left\langle r_{0}, d_{0}\right\rangle, P_{1}:\left\langle r_{1}+3 N-k, d_{1}\right\rangle$ \\
\hline $0 \leq k \leq N$ & 2 & $P_{0}{ }^{t} P_{1}$ & $P_{0}:\left\langle r_{0}, d_{0}\right\rangle, P_{1}:\left\langle r_{1}+(N-k) \%(2 N), d_{1}\right\rangle$ \\
\hline $0 \leq k \leq N$ & 2 & $P_{0}{ }^{t} P_{1}$ & $P_{0}:\left\langle r_{0}+k+N, d_{0}\right\rangle, P_{1}:\left\langle r_{1}, d_{1}\right\rangle$ \\
\hline$N \leq k \leq 2 N-1$ & 2 & $P_{0}{ }^{t} P_{1}$ & $P_{0}:\left\langle r_{0}+(k-N) \%(2 N), d_{0}\right\rangle, P_{1}:\left\langle r_{1}, d_{1}\right\rangle$ \\
\hline $0 \leq k \leq 2 N-1$ & 2 & $P_{0}{ }^{t} P_{1}$ & $P_{0}:\left\langle r_{0}+l, d_{0}\right\rangle, P_{1}:\left\langle r_{1}+(l-k+N) \%(2 N), d_{1}\right\rangle$ \\
& & & $(l=0, \ldots, N,(l-k+N) \%(2 N)=0, \ldots, N)$ \\
\hline $1 \leq k \leq 2 N-1$ & 4 & $P_{0}{ }^{t} P_{1}-{ }_{k} P_{2}{ }^{t} P_{3}$ & $P_{0}:\left\langle r_{0}, d_{0}\right\rangle$, \\
& & $P_{1}:\left\langle 2+l,\left(d_{0}+r_{0}+N-l-2\right) \%(2 N)\right\rangle$ \\
& & $P_{2}:\langle 2+(l+k-N) \%(2 N)$, \\
& & & $\left.\left(d_{0}+r_{0}-l-2\right) \%(2 N)\right\rangle$, \\
& & $P_{3}:\left\langle r_{1}, d_{1}\right\rangle$ \\
& & $(l=0, \ldots, N-1 ;$ \\
& & $(l+k+N) \%(2 N)=0, \ldots, N-1)$ \\
& & &
\end{tabular}

\section{Applications}

We have analyzed how shape features (PS-labels) and global structures (h/tconnections among primitive sequences) are transformed by various types of operations of T1 and T2. In this section, based on the transformation laws explored in Section 3, we describe some applications of the analysis to (a) the automatic induction of class descriptions from data, (b) the deduction of all the possible structural descriptions from the class descriptions, and (c) the examination of the status of conflicts among the class descriptions. 


\subsection{Automatic Construction of Class Descriptions}

The automatic construction of class descriptions from data can be considered as a problem of creating inductively, from the given data set, some shape representation that tolerates certain types of shape transformation. In particular, the transformations $\mathrm{T} 1$ and $\mathrm{T} 2$ addressed in this paper represent some typical shape deformations of handwritten characters. Therefore, we need to address the problem of generalizing shape descriptions that can be transformed via transformations T1 and T2.

However, shape deformations caused by $\mathrm{T} 1$ and $\mathrm{T} 2$ change topology and global features, and therefore, it is difficult to design an algorithm for generalizing structural descriptions into class descriptions. The solution to this problem is to explore complete a priori knowledge of the structural transformations caused by $\mathrm{T} 1$ and $\mathrm{T} 2$. Generalization is performed by comparing structural descriptions of two classes and finding out a relevant component correspondence by consistent labeling algorithms along with the high-level models of the transformations presented in Section 3 [3].

\subsection{Generation of Structural Descriptions from Classes}

For the character $\sigma$, suppose that we are given the class description $\sigma=\left(M_{\sigma}\right.$, $\left.\pi_{\sigma}, T_{\sigma}, \Gamma_{\sigma}\right)$, where $M_{\sigma}$ is the number of primitive sequences, $\pi(i)\left(i=1, \ldots, M_{\sigma}\right)$ is the set of eligible PS-labels for the primitive sequence $i, T_{\sigma}$ is a list of possible stroke connections representing T1 and T2, and $\Gamma_{\sigma}$ is the set of h/t-connections among primitive sequences. Let $S_{\sigma}=\left\{\left(l_{1}, \ldots, l_{M_{\sigma}}\right) \mid l_{i} \in \pi(i), i=1, \ldots, M_{\sigma}\right\}$ be the set of all the possible combinations of PS-labels of primitive sequences. For each $s \in S_{\sigma}$, by applying the stroke connections in $T_{\sigma}$ recursively along with the transformation laws shown in the Theorems [4], we can obtain all the possible structural descriptions from the class description $\sigma$. For instance, in the class description of " 3 ", Example 3 and Fig. 3 show all the possible structural descriptions (twelve, in total) for $s=(\langle 4,0\rangle,\langle 4,3\rangle) \in S_{3}$ along with stroke connections $T_{3}$. (The structural descriptions obtained by the transformation T1 are included by the ones obtained by T2.) Table 1 shows the number of structural descriptions deduced from some class descriptions for digits [4].

Table 1. Number of structural descriptions deduced from the class descriptions for digits.

\begin{tabular}{ccccccccccc}
\hline Character & 0 & 1 & 2 & 3 & 4 & 5 & 6 & 7 & 8 & 9 \\
\hline \#Descriptions & 16 & 4 & 66 & 403 & 444 & 316 & 18 & 929 & 2292 & 191 \\
\hline
\end{tabular}




\subsection{Conflicts among Class Descriptions}

From the sets of structural descriptions deduced from the class descriptions, we can easily find intersections among these sets by some relational matching procedures such as consistent labeling algorithms. Elements in the intersection of the two sets represent conflicts between the two class descriptions. Table 2 shows the number of structural descriptions in common with each pair of class descriptions. In particular, we should note that there are a number of conflicts between the class descriptions for " 4 " and " 7 ." The structural descriptions ignore metric information, and therefore, there are many instances of " 4 " and " 7 " that have the same descriptions as shown in Fig. 4. Most conflicts can be resolved by incorporating some geometrical or statistical information into the structural descriptions.

Table 2. Number of conflicts between two classes.

\begin{tabular}{|c|c|c|c|c|c|}
\hline \multicolumn{6}{|c|}{ 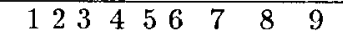 } \\
\hline 30 & 50 & 008 & 2 & 0 & \\
\hline 1 & 01 & 000 & 0 & 0 & \\
\hline 2 & & 1228 & 12 & 0 & 11 \\
\hline & & 1405 & 33 & 5 & \\
\hline & & 01 & 144 & 20 & 63 \\
\hline & & 0 & 0 & 2 & \\
\hline & & & & 0 & 3 \\
\hline & & & & & \\
\hline & & & & & 12 \\
\hline
\end{tabular}
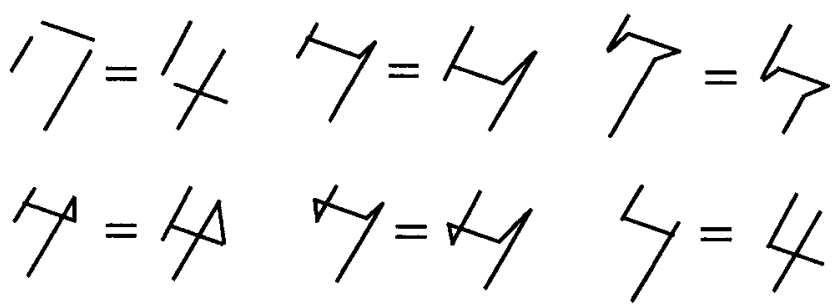

Fig. 4. Examples of the patterns of " 4 " and " 7 " that have the identical structural descriptions.

On the other hand, the analysis of metamorphosis of character shapes (transformation of an instance of a class into an instance of the other class via some 
transformation) has been an open problem in pattern recognition. An essential problem in handwriting recognition is how to cope with the complex shape deformation, and therefore, the modeling of the deformation and metamorphosis is a key to breaking through the difficulties in handwriting recognition. We have now a table of conflicts that has been deduced from the class description based on the structural model of shape deformation and the table well represents metamorphosis of characters. A possible extension of this work is to analyze the metamorphosis of characters by incorporating more information into the structural model presented in this paper.

\section{Conclusion}

Structural deformation caused by discontinuous transformations is an intractable problem in shape analysis and description. Structural descriptions depend on the topological structure of the shape, and therefore, they are sensitive to discontinuous transformations which change the topology of the shape. Because of the difficulties, there have been few systematic studies for analyzing and modeling structural deformations caused by discontinuous transformations. In this paper, as a first step for overcoming the difficulties, we have given an extensive analysis of structural deformations of curves due to some particular types of discontinuous transformations. Some systematic transformation laws have been obtained based on the shape description by Nishida and Mori [5], Nishida [1], [2]. We have also mentioned some applications of the analysis.

\section{References}

1. H. Nishida, "Structural feature extraction using multiple bases," Computer Vision and Image Understanding, vol. 62, no. 1, pp. 78-89, 1995.

2. H. Nishida, "Curve description based on directional features and quasi-convexity/ concavity," Pattern Recognition, vol. 28, no. 7, 1995.

3. H. Nishida, "Automatic construction of structural models incorporating discontinuous transformations," IEEE Trans. Pattern Analysis and Machine Intelligence, vol. 18 , no. $4,1996$.

4. H. Nishida, "A structural model of curve deformation by discontinuous transformations," Graphical Models and Image Processing, vol. 58, no. 2, 1996.

5. H. Nishida and S. Mori, "Algebraic description of curve structure," IEEE Trans. Pattern Analysis and Machine Intelligence, vol. 14, no. 5, pp. 516-533, 1992. 